\title{
Sound Transmission in a Duct with Sudden Area Expansion, Extended Inlet, and Lined Walls in Overlapping Region
}

\begin{abstract}
Ahmet Demir
Engineering Faculty, Department of Mechatronics, Karabuk University, 78100 Karabuk, Turkey

Correspondence should be addressed to Ahmet Demir; ademir@karabuk.edu.tr

Received 19 July 2016; Accepted 26 October 2016

Academic Editor: Toru Otsuru

Copyright (C) 2016 Ahmet Demir. This is an open access article distributed under the Creative Commons Attribution License, which permits unrestricted use, distribution, and reproduction in any medium, provided the original work is properly cited.

The transmission of sound in a duct with sudden area expansion and extended inlet is investigated in the case where the walls of the duct lie in the finite overlapping region lined with acoustically absorbent materials. By using the series expansion in the overlap region and using the Fourier transform technique elsewhere we obtain a Wiener-Hopf equation whose solution involves a set of infinitely many unknown expansion coefficients satisfying a system of linear algebraic equations. Numerical solution of this system is obtained for various values of the problem parameters, whereby the effects of these parameters on the sound transmission are studied.
\end{abstract}

\section{Introduction}

One can reduce the unwanted noise propagating along a duct by using a reactive or a dissipative silencer. In reactive silencers sudden area changes in cross-sectional area help to reduce the energy in the transmitted wave via internal reflections. Having a sudden area expansion together with a sudden area contraction simple expansion chambers works in accordance with this principle and is widely investigated in literature [1-4]. In further investigations it has been shown that the extension of inlet and outlet tubes into the expansion chamber increased the acoustic attenuation performance [57].

On the other hand, it has been proved that the treatment of the duct walls with an acoustically absorbent lining is another effective method in reducing unwanted noise [8]. Application of locally reacting linings or expansion chambers in ducts are efficient methods for noise reduction. These two methods were combined in [9] to discover transmission properties of a combination silencer consist of an expansion chamber whose walls are treated by acoustic liners and have been analysed by the author previously.

In this paper, the transmission of sound in an extended tube resonator whose walls are in overlapping region, where extended inlet and expanding duct walls overlap, are treated by locally reacting lining is investigated. So the main objective of this paper is to reveal the influence of the partial lining on the transmitted field and to present an alternative method of formulation. The method previously employed in $[10,11]$ consists of expanding the field in the overlap region into a series of complete set of orthogonal eigenfunctions and using the Fourier transform technique elsewhere. The problem is then reduced directly into a Wiener-Hopf equation whose solution involves a set of infinitely many unknown expansion coefficients satisfying an infinite systems of linear algebraic equations. Numerical solution to these systems is obtained for various values of the parameters of the problem such as the radii of the semi-infinite waveguides, the overlap length, and the impedance loading whereby the effects of these parameters on the transmitted field are presented graphically.

The time dependence is assumed to be $\exp (-i \omega t)$ with $\omega$ being the angular frequency and suppressed throughout.

\section{Materials and Methods}

Consider two opposite semi-infinite circular cylindrical waveguides of different radii with common longitudinal axis, say $z$, in a cylindrical polar coordinate system $(\rho, \phi, z)$. They occupy the regions $\rho=a$ and $z<l$ and $\rho=b>a$ and $z>0$, respectively, where $l$ represents the overlap length. These two waveguides are connected with a vertical wall at 
$a<\rho<b$ and $z=0$. The parts of the surfaces $r=a+0$ and $\rho=b-0$ lying in the overlap region $0<z<l$ of the waveguides and the vertical wall are assumed to be treated by acoustically absorbing linings which are characterized by constant but different surface admittances, say $\eta_{1}, \eta_{2}$, and $\eta_{3}$, respectively, while the remaining parts are perfectly rigid (see Figure 1). The waveguides are immersed in an inviscid and compressible stationary fluid of density $\tilde{\rho}_{0}$ and sound speed $c$. A plane sound wave is incident from the positive $z$ direction, through the waveguide of radius $\rho=a$. From the symmetry of the geometry of the problem and the incident field the acoustic field everywhere will be independent of the $\phi$ coordinate. We shall therefore introduce a scalar potential $u(\rho, z)$ which defines the acoustic pressure and velocity by $p=i \omega \widetilde{\rho}_{0} u$ and $\mathbf{v}=\operatorname{grad} u$, respectively.

Let the incident field be given by

$$
u^{i}=\exp (i k z)
$$

where $k=\omega / c$ denotes the wave number. For the sake of analytical convenience we will assume that the surrounding medium is slightly lossy and $k$ has a small positive imaginary part. The lossless case can be obtained by letting $\operatorname{Im} k \rightarrow 0$ at the end of the analysis.

The total field $u^{T}(\rho, z)$ can be written as

$$
u^{T}(\rho, z)= \begin{cases}u_{1}(\rho, z)+u^{i}(\rho, z), & \rho \in(0, a), z \in(-\infty, \infty), \\ u_{2}^{(1)}(\rho, z)[\mathscr{H}(z)-\mathscr{H}(z-l)]+u_{2}^{(2)}(\rho, z) \mathscr{H}(z-l), & \rho \in(a, b), \quad z \in(0, \infty) .\end{cases}
$$

$u_{1}(\rho, z)$ and $u_{2}^{(j)}(\rho, z)(j=1,2)$ denote the scattered fields which satisfy the Helmholtz equation

$$
\left[\frac{1}{\rho} \frac{\partial}{\partial \rho}\left(\rho \frac{\partial}{\partial \rho}\right)+\frac{\partial^{2}}{\partial z^{2}}+k^{2}\right]\left[\begin{array}{c}
u_{1}(\rho, z) \\
u_{2}^{(j)}(\rho, z)
\end{array}\right]=0
$$

$$
j=1,2
$$

and are to be determined with the help of the following boundary and continuity relations:

$$
\begin{gathered}
\frac{\partial}{\partial \rho} u_{1}(a, z)=0, \quad z<l, \\
\frac{\partial}{\partial z} u_{2}^{(1)}(\rho, 0)=0, \\
a<\rho<b, \\
{\left[i k \eta_{1}+\frac{\partial}{\partial \rho}\right] u_{2}^{(1)}(a, z)=0,} \\
0<z<l, \\
{\left[i k \eta_{2}-\frac{\partial}{\partial \rho}\right] u_{2}^{(1)}(b, z)=0,} \\
{\left[i k \eta_{3}+\frac{\partial}{\partial z}\right] u_{2}^{(1)}(\rho, 0)=0,} \\
a<\rho<l, \\
\frac{\partial}{\partial \rho} u_{2}^{(2)}(b, z)=0, \quad z>l, \\
u_{2}^{(1)}(\rho, l)-u_{2}^{(2)}(\rho, l)=0,
\end{gathered}
$$

$$
\begin{aligned}
\frac{\partial}{\partial z} u_{2}^{(1)}(\rho, l)-\frac{\partial}{\partial z} u_{2}^{(2)}(\rho, l)=0, & \\
& a<\rho<b, \\
u_{1}(a, z)+u_{i}(a, z)-u_{2}^{(2)}(a, z)=0, & z>l, \\
\frac{\partial}{\partial \rho} u_{1}(a, z)+\frac{\partial}{\partial \rho} u_{i}(a, z)-\frac{\partial}{\partial \rho} u_{2}^{(2)}(a, z)=0, & z>l .
\end{aligned}
$$

In addition to these boundary and continuity relations one has to take into account the following radiation and edge conditions to ensure the uniqueness of the mixed boundary value problem stated by (3) and (4a) $-(4 \mathrm{j})$ :

$$
\begin{aligned}
u & \sim \frac{e^{i k r}}{r}, \quad r=\sqrt{\rho^{2}+z^{2}} \longrightarrow \infty, \\
u^{T}(\rho, z) & =O(1), \quad z \longrightarrow l, \\
\frac{\partial}{\partial \rho} u^{T}(\rho, z) & =O\left((z-l)^{-1 / 2}\right), \quad z \longrightarrow l .
\end{aligned}
$$

2.1. The Wiener-Hopf Equations. Consider the Fourier transform of the Helmholtz equation satisfied by the scattered field $u_{1}(\rho, z)$ in the region $\rho<a$ for $z \in(-\infty, \infty)$; namely,

$$
\left[\frac{1}{\rho} \frac{\partial}{\partial \rho}\left(\rho \frac{\partial}{\partial \rho}\right)+K^{2}(\alpha)\right] F(\rho, \alpha)=0
$$

where $F(\rho, \alpha)$ is the Fourier transform of the field $u_{1}(\rho, z)$ defined to be

$$
\begin{aligned}
F(\rho, \alpha) & =\int_{-\infty}^{\infty} u_{1}(\rho, z) e^{i \alpha z} d z \\
& =e^{i \alpha l}\left[F_{+}(\rho, \alpha)+F_{-}(\rho, \alpha)\right]
\end{aligned}
$$


with

$$
\begin{aligned}
& F_{-}(\rho, \alpha)=\int_{-\infty}^{l} u_{1}(\rho, z) e^{i \alpha(z-l)} d z, \\
& F_{+}(\rho, \alpha)=\int_{l}^{\infty} u_{1}(\rho, z) e^{i \alpha(z-l)} d z .
\end{aligned}
$$

Owing to the analytical properties of Fourier integrals, $F_{+}(\rho, \alpha)$ and $F_{-}(\rho, \alpha)$ are regular functions in the upper halfplane $\operatorname{Im} \alpha>\operatorname{Im}(-k)$ and in the lower half-plane $\operatorname{Im} \alpha<\operatorname{Im} k$, respectively. The solution of (7) reads

$$
F(\rho, \alpha)=-A(\alpha) \frac{J_{0}(K \rho)}{K(\alpha) J_{1}(K a)},
$$

where $A(\alpha)$ is a spectral coefficient to be determined and $K(\alpha)$ is the square-root function

$$
K(\alpha)=\sqrt{k^{2}-\alpha^{2}}
$$

which is defined in the complex $\alpha$-plane cut as shown in Figure 2 such that $K(0)=k$. Consider now the Fourier transform of (4a); namely,

$$
\dot{F}_{-}(a, \alpha)=0 .
$$

The differentiation of (9) with respect to $\rho$ and putting $\rho=a$ gives

$$
e^{i \alpha l} \dot{F}_{+}(a, \alpha)=A(\alpha) .
$$

Substituting (12) into (9) yields

$$
F_{+}(\rho, \alpha)=-\dot{F}_{+}(a, \alpha) \frac{J_{0}(K \rho)}{K(\alpha) J_{1}(K a)}-F_{-}(\rho, \alpha) .
$$

In the region $a<\rho<b$ the field $u_{2}^{(2)}(\rho, z)$ satisfies the Helmholtz equation for $z \in(l, \infty)$ as denoted in (3). The Fourier transform of this equation for the region in question is

$$
\begin{aligned}
& {\left[\frac{1}{\rho} \frac{\partial}{\partial \rho}\left(\rho \frac{\partial}{\partial \rho}\right)+K^{2}(\alpha)\right] G_{+}(\rho, \alpha)} \\
& \quad=f(\rho)-i \alpha g(\rho),
\end{aligned}
$$

where

$$
\begin{aligned}
& f(\rho)=\frac{\partial}{\partial z} u_{2}^{(2)}(\rho, l), \\
& g(\rho)=u_{2}^{(2)}(\rho, l) .
\end{aligned}
$$

In (14), $G_{+}(\rho, \alpha)$ is a regular function in the upper half of the complex $\alpha$-plane which is defined as

$$
G_{+}(\rho, \alpha)=\int_{l}^{\infty} u_{2}^{(2)}(\rho, z) e^{i \alpha(z-l)} d z
$$

Particular solutions to (14) can be found easily by using Green's function which satisfies the Helmholtz equation

$$
\left[\frac{1}{\rho} \frac{\partial}{\partial \rho}\left(\rho \frac{\partial}{\partial \rho}\right)+K^{2}(\alpha)\right] \mathscr{G}(\rho, \alpha)=0
$$

with the following conditions:

$$
\rho \neq t, \rho, t \in(a, b)
$$

$$
\begin{aligned}
\mathscr{G}(t+0, t, \alpha) & =\mathscr{G}(t-0, t, \alpha) \\
\frac{\partial}{\partial \rho} \mathscr{G}(t+0, t, \alpha)-\frac{\partial}{\partial \rho} \mathscr{G}(t-0, t, \alpha) & =\frac{1}{t} \\
\frac{\partial}{\partial \rho} \mathscr{G}(b, t, \alpha) & =0 \\
\frac{\partial}{\partial \rho} \mathscr{G}(a, t, \alpha) & =0
\end{aligned}
$$

The solution is

$$
\mathscr{G}(\rho, t, \alpha)=\frac{1}{K^{2}(\alpha) M(\alpha)} Q(\rho, t, \alpha)
$$

with

$$
\begin{aligned}
Q(\rho, t, \alpha) & =\frac{\pi}{2} \begin{cases}{\left[J_{0}(K \rho) K Y_{1}(K a)-K J_{1}(K a) Y_{0}(K \rho)\right]\left[J_{0}(K t) K Y_{1}(K b)-K J_{1}(K b) Y_{0}(K t)\right],} & a \leq \rho \leq t, \\
{\left[J_{0}(K \rho) K Y_{1}(K b)-K J_{1}(K b) Y_{0}(K \rho)\right]\left[J_{0}(K t) K Y_{1}(K a)-K J_{1}(K a) Y_{0}(K t)\right],} & t \leq \rho \leq b,\end{cases} \\
M(\alpha) & =\left[J_{1}(K a) Y_{1}(K b)-J_{1}(K b) Y_{1}(K a)\right] .
\end{aligned}
$$

Together with the boundary condition (4f) the solution can be written as

$$
\begin{aligned}
G_{+} & (\rho, \alpha)=\frac{1}{K^{2}(\alpha) M(\alpha)}\{B(\alpha) \\
\cdot & {\left[J_{0}(K \rho) K Y_{1}(K b)-Y_{0}(K \rho) K J_{1}(K b)\right] }
\end{aligned}
$$

$$
\left.+\int_{a}^{b}[f(t)-i \alpha g(t)] Q(t, \rho, \alpha) t d t\right\} .
$$

Here, $B(\alpha)$ is a spectral coefficient to be determined. 


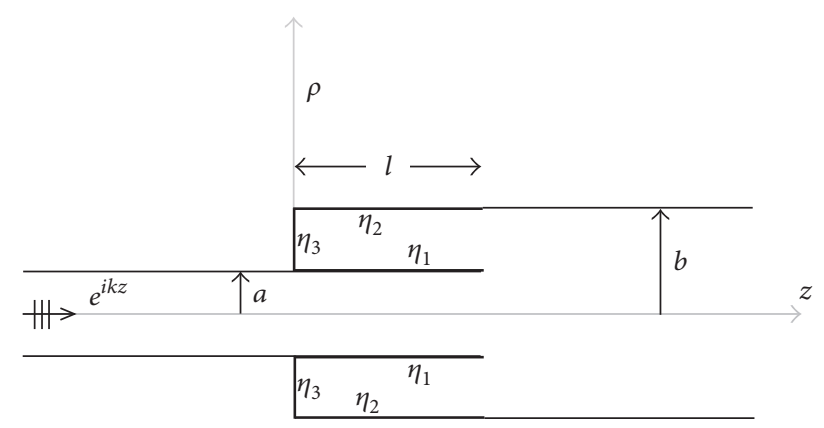

FIgURE 1: Geometry of the problem.

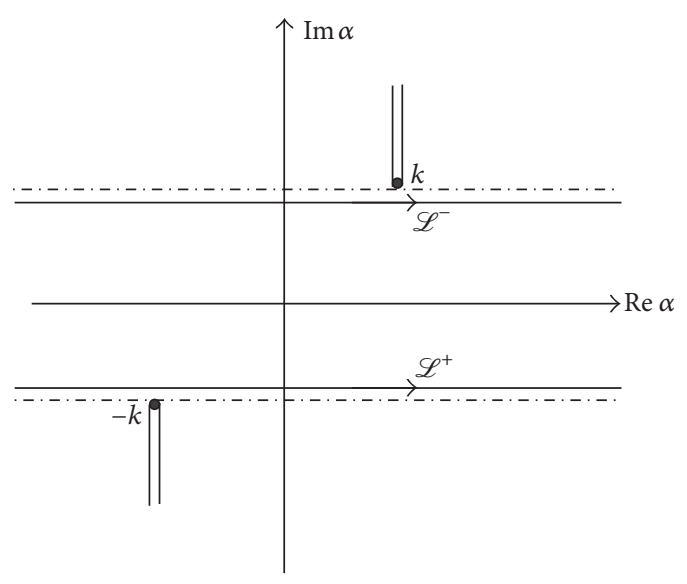

FIGURE 2: Complex $\alpha$-plane.

The continuity relation in $(4 j)$ requires

$$
\dot{G}_{+}(a, \alpha)=\dot{F}_{+}(a, \alpha) .
$$

$B(\alpha)$ can be solved uniquely from (22) as

$$
B(\alpha)=-\dot{F}_{+}(a, \alpha)
$$

The substitution of (23) into (21) gives

$$
\begin{gathered}
G_{+}(\rho, \alpha)=\frac{1}{K^{2}(\alpha) M(\alpha)}\left\{-\dot{F}_{+}(a, \alpha)\right. \\
\cdot\left[J_{0}(K \rho) K Y_{1}(K b)-Y_{0}(K \rho) K J_{1}(K b)\right] \\
\left.+\int_{a}^{b}[f(t)-i \alpha g(t)] Q(t, \rho, \alpha) t d t\right\} .
\end{gathered}
$$

Although the left-hand side of (24) is regular in the halfplane $\operatorname{Im}(\alpha)>\operatorname{Im}(-k)$, the regularity of the right hand side is violated by the presence of simple poles lying at the upper half-plane, namely, at $\alpha=\alpha_{m}\left(\operatorname{Im}\left(\alpha_{m}\right)>\operatorname{Im}(k)\right)$ with

$$
\begin{aligned}
& K^{2}\left(\alpha_{m}\right)\left[J_{1}\left(K_{m} a\right) Y_{1}\left(K_{m} b\right)-J_{1}\left(K_{m} b\right) Y_{1}\left(K_{m} a\right)\right] \\
& \quad=0, \quad K_{m}=K\left(\alpha_{m}\right), m=0,1,2, \ldots
\end{aligned}
$$

In order to provide regularity of the right hand side of (24) in the upper half of the $\alpha$-plane, these poles must be eliminated by imposing that their residues are zero. This gives

$$
\begin{aligned}
\dot{F}_{+}(a, k) & =\frac{1}{\pi}\left[f_{0}-i k g_{0}\right] \frac{a^{2}-b^{2}}{a b}, \\
\dot{F}_{+}\left(a, \alpha_{m}\right) & =\frac{1}{\pi}\left[f_{m}-i \alpha_{m} g_{m}\right] \frac{J_{1}^{2}\left(K_{m} a\right)-J_{1}^{2}\left(K_{m} b\right)}{J_{1}\left(K_{m} a\right) J_{1}\left(K_{m} b\right)}
\end{aligned}
$$

for $m=0,1,2, \ldots$. Here, $f_{m}$ and $g_{m}$ 's are the expansion coefficients of the functions $f(\rho)$ and $g(\rho)$, respectively, which may be represented trough the following complete sets of orthogonal functions

$$
\begin{aligned}
& {\left[\begin{array}{l}
f(\rho) \\
g(\rho)
\end{array}\right]=\frac{2}{\pi b}\left[\begin{array}{l}
f_{0} \\
g_{0}
\end{array}\right]} \\
& \quad+\sum_{m=1}^{\infty}\left[\begin{array}{l}
f_{m} \\
g_{m}
\end{array}\right]\left[J_{0}\left(K_{m} \rho\right) K_{m} Y_{1}\left(K_{m} b\right)\right. \\
& \left.\quad-K_{m} J_{1}\left(K_{m} b\right) Y_{0}\left(K_{m} \rho\right)\right] .
\end{aligned}
$$

Using the continuity relation (4i) together with (24) and taking into account (13) give

$$
\begin{aligned}
& \frac{\dot{F}_{+}(a, \alpha)}{K^{2}(\alpha)} \frac{J_{1}(K b)}{\pi M(\alpha) J_{1}(K a)}+\frac{a}{2} F_{-}(a, \alpha)=\frac{1}{2} \\
& \cdot \frac{1}{K^{2}(\alpha) M(\alpha)} \int_{a}^{b}[f(t)-i \alpha g(t)] \\
& \cdot\left[J_{0}(K t) K Y_{1}(K b)-K J_{1}(K b) Y_{0}(K t)\right] t d t-\frac{a}{2} \\
& \cdot \frac{e^{i k l}}{i(\alpha+k)}, \\
& \frac{\dot{F}_{+}(a, \alpha)}{K^{2}(\alpha)} N(\alpha)+\frac{a}{2} F_{-}(a, \alpha)=\frac{b}{\pi a} \frac{\left[f_{0}-i \alpha g_{0}\right]}{k^{2}-\alpha^{2}}+\frac{1}{\pi} \\
& \cdot \sum_{m=1}^{\infty} \frac{\left[f_{m}-i \alpha g_{m}\right]}{\alpha_{m}^{2}-\alpha^{2}} \frac{J_{1}\left(K_{m} b\right)}{J_{1}\left(K_{m} a\right)}-\frac{a}{2} \frac{e^{i k l}}{i(\alpha+k)}
\end{aligned}
$$

which is the Wiener-Hopf equation to be solved through classical procedures. Here, $N(\alpha)$ stands for

$$
N(\alpha)=\frac{J_{1}(K b)}{\pi M(\alpha) J_{1}(K a)} .
$$

The final solution of the $\mathrm{W}-\mathrm{H}$ equation is determined to be

$$
\begin{gathered}
\frac{\dot{F}_{+}(a, \alpha)}{(k+\alpha)} N_{+}(\alpha)=-\frac{a}{\pi b} \frac{\left[f_{0}+i k g_{0}\right]}{(k+\alpha) N_{+}(k)}+\frac{1}{\pi} \\
\cdot \sum_{m=1}^{\infty} \frac{\left[f_{m}+i \alpha_{m} g_{m}\right]}{2 \alpha_{m}\left(\alpha_{m}+\alpha\right)} \frac{J_{1}\left(K_{m} b\right)}{J_{1}\left(K_{m} a\right)} \frac{k+\alpha_{m}}{N_{+}\left(\alpha_{m}\right)} \\
-\frac{k a e^{i k l}}{i(\alpha+k) N_{+}(k)},
\end{gathered}
$$


where $N_{ \pm}(\alpha)$ are the split functions resulting from the Wiener-Hopf factorization of $N(\alpha)$ as

$$
N(\alpha)=N_{+}(\alpha) N_{-}(\alpha) .
$$

Their explicit expressions are given in [12] as

$$
\begin{aligned}
& N_{+}(\alpha)=[\pi \\
& \left.\cdot \frac{J_{1}(k a)}{J_{1}(k b)}\left[J_{1}(k a) Y_{1}(k b)-J_{1}(k b) Y_{1}(k a)\right]\right]^{-1 / 2} \\
& \cdot e^{-\alpha \chi} \prod_{n=0}^{\infty} \frac{\left(1+\alpha / \sqrt{k^{2}-\left(j_{n} / b\right)^{2}}\right)}{\left(1+\alpha / \sqrt{k^{2}-\left(j_{n} / a\right)^{2}}\right)\left(1+\alpha / \alpha_{n}\right)} .
\end{aligned}
$$

Here $j_{n}$ 's are the roots of the Bessel function of the first kind

$$
\begin{aligned}
J_{1}\left(j_{n}\right) & =0, \quad n=0,1, \ldots, \\
\chi & =\frac{i}{\pi}[b \ln b-a \ln a-(b-a) \ln (b-a)]
\end{aligned}
$$

with

$$
N_{-}(\alpha)=N_{+}(-\alpha)
$$

2.2. Determination of the Unknown Coefficients. The field in the region $a<\rho<b, z \in(0, l)$ can be expressed is terms of the waveguide normal modes as

$$
\begin{aligned}
& u_{2}^{(1)}(\rho, z) \\
& \quad=\sum_{n=0}^{\infty} a_{n}\left[e^{i \beta_{n} z}-P_{n} e^{-i \beta_{n} z}\right]\left[J_{0}\left(\xi_{n} \rho\right)-R_{n} Y_{0}\left(\xi_{n} \rho\right)\right]
\end{aligned}
$$

with

$$
\begin{aligned}
P_{n} & =\frac{i k \eta_{3}+i \beta_{n}}{i k \eta_{3}-i \beta_{n}}, \\
R_{n} & =\frac{i k \eta_{1} J_{0}\left(\xi_{n} a\right)-\xi_{n} J_{1}\left(\xi_{n} a\right)}{i k \eta_{1} Y_{0}\left(\xi_{n} a\right)-\xi_{n} Y_{1}\left(\xi_{n} a\right)} \\
& =\frac{i k \eta_{2} J_{0}\left(\xi_{n} b\right)+\xi_{n} J_{1}\left(\xi_{n} b\right)}{i k \eta_{2} Y_{0}\left(\xi_{n} b\right)+\xi_{n} Y_{1}\left(\xi_{n} b\right)} .
\end{aligned}
$$

In (34), $\xi_{n}$ 's are the roots of the following equation:

$$
\begin{aligned}
& \frac{i k \eta_{1} J_{0}\left(\xi_{n} a\right)-\xi_{n} J_{1}\left(\xi_{n} a\right)}{i k \eta_{1} Y_{0}\left(\xi_{n} a\right)-\xi_{n} Y_{1}\left(\xi_{n} a\right)} \\
& -\frac{i k \eta_{2} J_{0}\left(\xi_{n} b\right)+\xi_{n} J_{1}\left(\xi_{n} b\right)}{i k \eta_{2} Y_{0}\left(\xi_{n} b\right)+\xi_{n} Y_{1}\left(\xi_{n} b\right)}=0
\end{aligned}
$$

while $\beta_{n}$ 's are defined as

$$
\beta_{n}=\sqrt{k^{2}-\xi_{n}^{2}}
$$

Taking into account (27) and (34), the continuity relations $(4 \mathrm{~g})$ and $(4 \mathrm{~h})$ can be written in the following form:

$$
\begin{aligned}
& \frac{2}{\pi b}\left[f_{0}+i \alpha g_{0}\right]+\sum_{m=1}^{\infty}\left[f_{m}+i \alpha g_{m}\right] \\
& \cdot\left[J_{0}\left(K_{m} \rho\right) K_{m} Y_{1}\left(K_{m} b\right)\right. \\
& \left.\quad-K_{m} J_{1}\left(K_{m} b\right) Y_{0}\left(K_{m} \rho\right)\right]=i \sum_{n=0}^{\infty} a_{n}\left[\left(\alpha+\beta_{n}\right) e^{i \beta_{n} l}\right. \\
& \left.\quad-P_{n}\left(\alpha-\beta_{n}\right) e^{-i \beta_{n} l}\right]\left[J_{0}\left(\xi_{n} \rho\right)-R_{n} Y_{0}\left(\xi_{n} \rho\right)\right] .
\end{aligned}
$$

Multiplying (38) by $2 \rho / \pi b$ and $\left[J_{0}\left(K_{m} \rho\right) K_{m} Y_{1}\left(K_{m} b\right)-\right.$ $\left.K_{m} J_{1}\left(K_{m} b\right) Y_{0}\left(K_{m} \rho\right)\right] \rho$, respectively, and then integrating over $\rho$ from $a$ to $b$ read

$$
\begin{aligned}
& {\left[f_{0}+i \alpha g_{0}\right]} \\
& =\frac{i}{S_{0}} \sum_{n=0}^{\infty} a_{n}\left[\left(\alpha+\beta_{n}\right) e^{i \beta_{n} l}-P_{n}\left(\alpha-\beta_{n}\right) e^{-i \beta_{n} l}\right] \triangle_{0 n} \\
& {\left[f_{m}+i \alpha g_{m}\right]} \\
& =\frac{i}{S_{m}} \sum_{n=0}^{\infty} a_{n}\left[\left(\alpha+\beta_{n}\right) e^{i \beta_{n} l}-P_{n}\left(\alpha-\beta_{n}\right) e^{-i \beta_{n} l}\right] \triangle_{m n}
\end{aligned}
$$

with

$$
\begin{gathered}
\triangle_{0 n}=\frac{2}{\pi \xi_{n}}\left\{\frac{a}{b}\left[J_{1}\left(\xi_{n} a\right)-R_{n} Y_{1}\left(\xi_{n} a\right)\right]-\left[J_{1}\left(\xi_{n} b\right)\right.\right. \\
\left.\left.-R_{n} Y_{1}\left(\xi_{n} b\right)\right]\right\}, \\
\triangle_{m n}=\frac{2 \xi_{n}}{\pi\left(\xi_{n}^{2}-K_{m}^{2}\right)}\left\{\frac { J _ { 1 } ( K _ { m } b ) } { J _ { 1 } ( K _ { m } a ) } \left[J_{1}\left(\xi_{n} a\right)\right.\right. \\
\left.\left.-R_{n} Y_{1}\left(\xi_{n} a\right)\right]-\left[J_{1}\left(\xi_{n} b\right)-R_{n} Y_{1}\left(\xi_{n} b\right)\right]\right\}
\end{gathered}
$$

where $S_{0}$ and $S_{m}$ stand for

$$
\begin{aligned}
& S_{0}=\frac{2}{\pi^{2}} \frac{a^{2}-b^{2}}{b^{2}}, \\
& S_{m}=\frac{2}{\pi^{2}} \frac{J_{1}^{2}\left(K_{m} a\right)-J_{1}^{2}\left(K_{m} b\right)}{J_{1}^{2}\left(K_{m} a\right)} .
\end{aligned}
$$


Using the W-H solution (30) together with (26a) and (26b) we obtain a set of linear algebraic equations in terms of the unknown coefficients $f_{m}$ and $g_{m}$.

$$
\begin{aligned}
& \frac{\pi}{2} \frac{b}{a}\left[f_{0}-i k g_{0}\right] S_{0} N_{+}(k)=\frac{b}{\pi a} \frac{\left[f_{0}+i k g_{0}\right]}{N_{+}(k)}+\frac{k}{\pi} \\
& \cdot \sum_{m=1}^{\infty} \frac{\left[f_{m}+i \alpha_{m} g_{m}\right]}{\alpha_{m} N_{+}\left(\alpha_{m}\right)} \frac{J_{1}\left(K_{m} b\right)}{J_{1}\left(K_{m} a\right)}-\frac{k a e^{i k l}}{i N_{+}(k)}, \\
& \frac{\pi}{2}\left[f_{r}-i \alpha_{r} g_{r}\right] \frac{J_{1}\left(K_{r} a\right)}{J_{1}\left(K_{r} b\right)} S_{r} N_{+}\left(\alpha_{r}\right)=\frac{b}{\pi a} \frac{\left[f_{0}+i k g_{0}\right]}{N_{+}(k)} \\
& +\frac{1}{\pi}\left(k+\alpha_{r}\right) \\
& \quad \sum_{m=1}^{\infty} \frac{\left[f_{m}+i \alpha_{m} g_{m}\right]}{2 \alpha_{m}\left(\alpha_{m}+\alpha_{r}\right)} \frac{J_{1}\left(K_{m} b\right)}{J_{1}\left(K_{m} a\right)} \frac{k+\alpha_{m}}{N_{+}\left(\alpha_{m}\right)} \\
& \quad-\frac{k a e^{i k l}}{i N_{+}(k)},
\end{aligned}
$$

and taking into account (39a) and (39b) we obtain now a set of equations to determine the unknown expansion coefficient $a_{n}$ as

$$
\begin{aligned}
- & i \frac{\pi}{2} \frac{b}{a} N_{+}(k) \sum_{n=0}^{\infty} a_{n}\left[\left(k-\beta_{n}\right) e^{i \beta_{n} l}-P_{n}\left(k+\beta_{n}\right) e^{-i \beta_{n} l}\right] \triangle_{0 n} \\
& =\frac{b}{\pi a} \frac{1}{N_{+}(k)} \frac{i}{S_{0}} \sum_{n=0}^{\infty} a_{n}\left[\left(k+\beta_{n}\right) e^{i \beta_{n} l}-P_{n}\left(k-\beta_{n}\right)\right. \\
& \left.\cdot e^{-i \beta_{n} l}\right] \triangle_{0 n}+i \frac{k}{\pi} \sum_{n=0}^{\infty} \sum_{m=1}^{\infty} a_{n}\left[\left(\alpha_{m}+\beta_{n}\right) e^{i \beta_{n} l}\right. \\
& \left.-P_{n}\left(\alpha_{m}-\beta_{n}\right) e^{-i \beta_{n} l}\right] \frac{\triangle_{m n}}{\alpha_{m} N_{+}\left(\alpha_{m}\right)} \frac{J_{1}\left(K_{m} b\right)}{S_{m} J_{1}\left(K_{m} a\right)} \\
& -\frac{k a e^{i k l}}{i N_{+}(k)}, \\
- & i \frac{\pi}{2} \frac{J_{1}\left(K_{r} a\right)}{J_{1}\left(K_{r} b\right)} N_{+}\left(\alpha_{r}\right) \sum_{n=0}^{\infty} a_{n}\left[\left(\alpha_{r}-\beta_{n}\right) e^{i \beta_{n} l}-P_{n}\left(\alpha_{r}\right.\right. \\
& \left.+\beta_{n}\right) e^{\left.-i \beta_{n} l\right]} \triangle_{m}=\frac{b}{\pi a} \frac{1}{N_{+}(k)} \frac{i}{S_{0}} \sum_{n=0}^{\infty} a_{n}\left[\left(k+\beta_{n}\right) e^{i \beta_{n} l}\right. \\
& -\frac{k a e^{i k l}}{i N_{+}(k)} \cdot \\
& \cdot \frac{\left(k+\alpha_{r}\right) \triangle_{m n}}{2 \alpha_{m}\left(\alpha_{m}+\alpha_{r}\right)} \frac{J_{1}\left(K_{m} b\right)}{S_{m} J_{1}\left(K_{m} a\right)} \frac{k+\alpha_{m}}{N_{+}\left(\alpha_{m}\right)} \\
& \sum_{n=0}^{\infty} \sum_{m=1}^{\infty} a_{n}\left[\left(\alpha_{m}+\beta_{n}\right) e^{i \beta_{n} l}-P_{n}\left(\alpha_{m}-\beta_{n}\right) e^{-i \beta_{n} l}\right] \\
& \left(k \triangle_{0 n}+\frac{i}{\pi}\right.
\end{aligned}
$$

2.3. Reflected and Transmitted Fields. According to (8a), the scattered field in the region $0<\rho<a$, that is, $u_{1}(\rho, z)$, can be obtained by taking the inverse Fourier transform of $F(\rho, \alpha)$. By considering (13) we write

$$
\begin{aligned}
& u_{1}(\rho, z) \\
& \quad=-\frac{1}{2 \pi} \int_{\mathscr{L}} \dot{F}_{+}(a, \alpha) \frac{J_{0}(K \rho)}{K(\alpha) J_{1}(K a)} e^{-i \alpha(z-l)} d \alpha .
\end{aligned}
$$

The evaluation of this integral for $z<l$ and $z>l$ will give us the reflected wave propagating backward in the inner cylinder and the transmitted wave, respectively.

For $z<l$, the integral is calculated by closing the contour in the upper half-plane and evaluating the residues contributions from the simple poles occurring at the zeros of $J_{1}(K a)$ lying in the upper $\alpha$-half-plane, namely, at $K a=$ $j_{n}$. The reflection coefficient $\mathscr{R}$ of the fundamental mode is defined as the complex coefficient multiplying the travelling wave term $\exp (-i k z)$ and is computed from the contribution of the first pole at $\alpha=k$. The result is

$$
\begin{aligned}
\mathscr{R}= & -\frac{e^{i 2 k l}}{\left[N_{+}(k)\right]^{2}}-\frac{i}{\pi} \frac{\left[f_{0}+i k g_{0}\right]}{k b\left[N_{+}(k)\right]^{2}} e^{i k l} \\
& +\frac{i}{\pi} \frac{e^{i k l}}{a N_{+}(k)} \sum_{m=1}^{\infty} \frac{\left[f_{m}+i \alpha_{m} g_{m}\right]}{\alpha_{m} N_{+}\left(\alpha_{m}\right)} \frac{J_{1}\left(K_{m} b\right)}{J_{1}\left(K_{m} a\right)} .
\end{aligned}
$$

The first term is the reflection coefficient related to the case where a semi-infinite rigid duct is inserted axially into a larger rigid tube of infinite length [12] whereas the second one is the correction term involving the effect of the impedances of the annular region and the overlap length.

Similarly, the transmission coefficient $\mathscr{T}$ of the fundamental mode which is defined as the complex coefficient of $\exp (i k z)$ is obtained by evaluating the integral in (44) for $z>l$. This integral is now computed by closing the contour in the lower half of the complex $\alpha$-plane. The pole of interest is at $\alpha=-k$ whose contribution gives

$$
(-1+\mathscr{T}) e^{i k z}+O\left(e^{i \sqrt{k^{2}-\left(j_{n} / b\right)^{2}} z}\right)
$$

with

$$
\mathscr{T}=\frac{a^{2}}{b^{2}}+\frac{i e^{-i k l}}{\pi k a}\left(\frac{b}{a}-\frac{a}{b}\right)\left[f_{0}+i k g_{0}\right]
$$

The first term in (46a) cancels out the incident wave in the region $\rho<a, z>l$, while the second is the transmission coefficient of the fundamental mode.

\section{Results and Discussion}

In this section in order to show the effects of the parameters like the length of the extended inlet $l$ and the surface admittance $\eta_{1,2,3}$ on the transmitted field, some numerical results showing variation of the transmission coefficient $\mathscr{T}$ with different parameters are presented. In all numerical calculations the solution of the infinite system of algebraic equations is obtained by truncating the infinite series at $N=$ 5 , since the transmission coefficient becomes insensitive for 


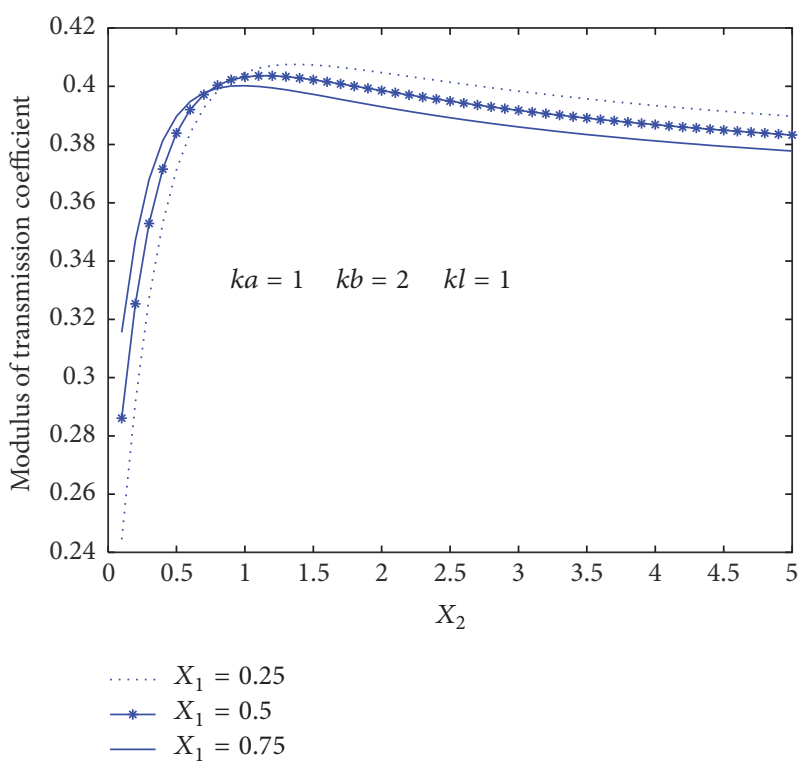

FIGURE 3: Transmission coefficient $\mathscr{T}$ versus the surface admittance $\eta_{2}=i X_{2}\left(X_{2}>0\right)$ for different values of $\eta_{1}=i X_{1}$.

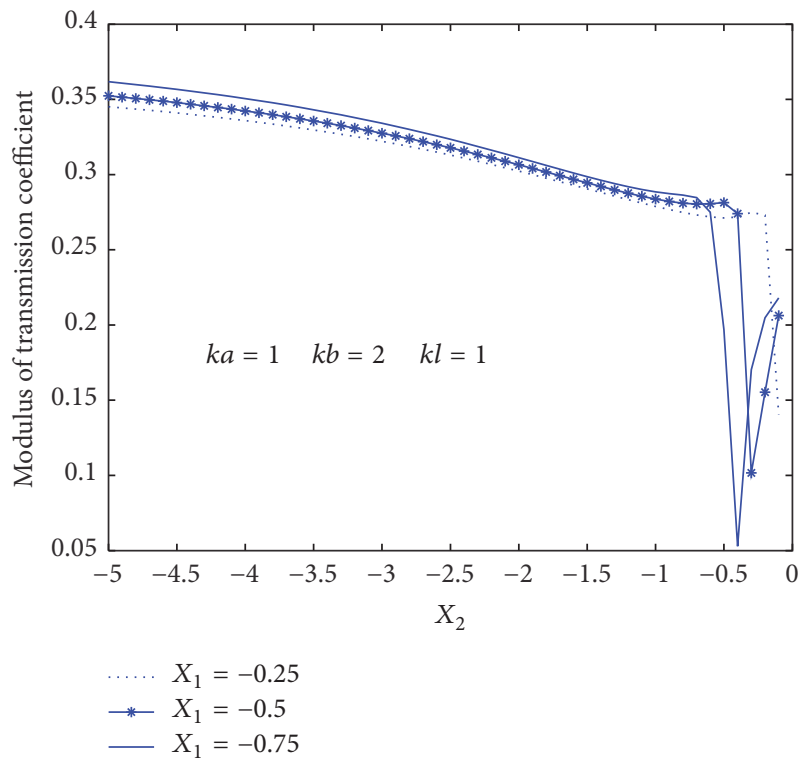

FIGURE 4: Transmission coefficient $\mathscr{T}$ versus the surface admittance $\eta_{2}=i X_{2}\left(X_{2}<0\right)$ for different values of $\eta_{1}=i X_{1}$.

$N>5$. We also limit ourselves with only imaginary values of surface admittance $\eta_{1,2}$ for simplicity.

In Figures 3 and 4, while the admittance $X_{2}>0$ of the lateral wall of the expanding duct increases the transmitted field is ascending until some value of $X_{2}$; then it starts to attenuate gradually. But for negative values of $X_{2}$ the attenuation is more visible especially around $-0.5<X_{2}<0$. For different values of $X_{1}$ not much but some decrease in the transmitted field is observed.

In Figure 5, an oscillatory behaviour is seen for increasing values of the extended inlet length $\mathrm{kl}$, but this behaviour is

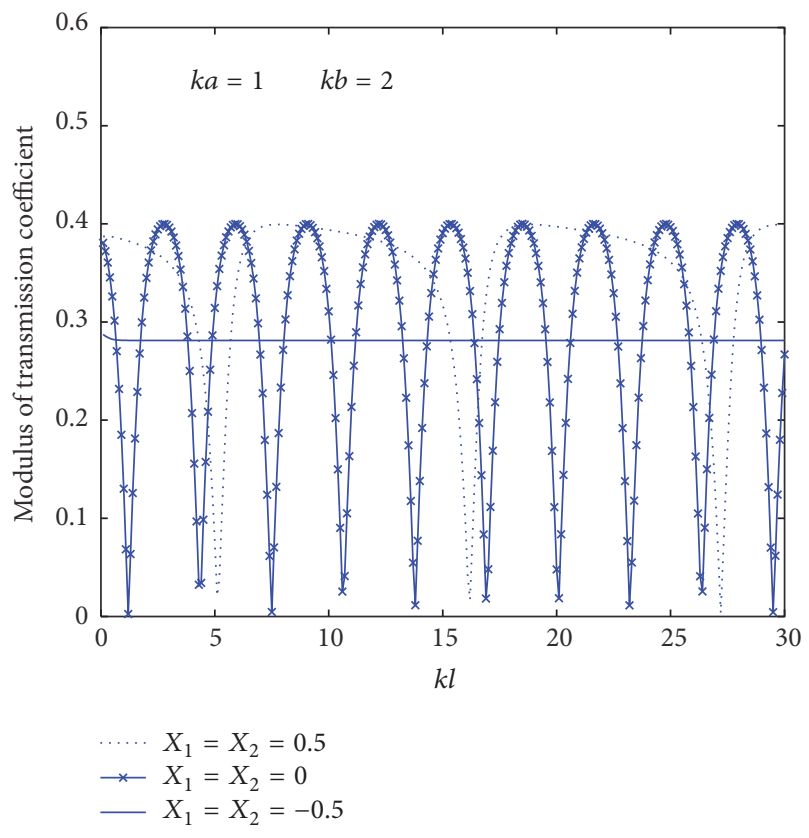

Figure 5: Transmission coefficient $\mathscr{T}$ versus the extended inlet length $k l$ for different values of $\eta_{1}=i X_{1}$ and $\eta_{2}=i X_{2}$.

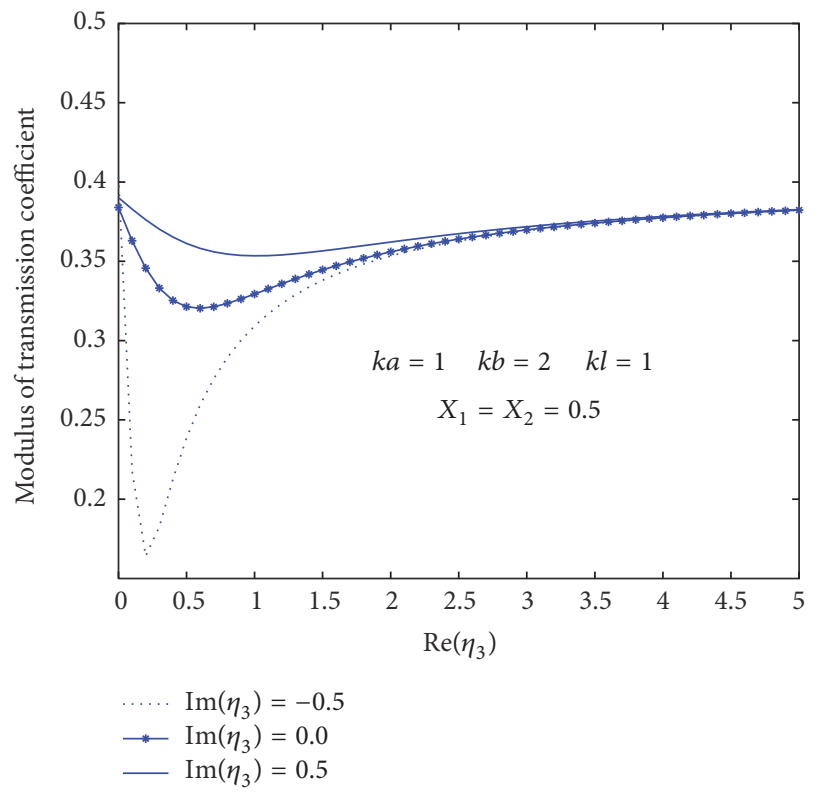

FIGURE 6: Transmission coefficient $\mathscr{T}$ versus the real part of $\eta_{3}$ for different values of $\operatorname{Im}\left(\eta_{3}\right)$.

broken for negative values of $X_{1}$ and $X_{2}$. From Figure 6 it is observed that the transmission does not alter as the real part of $\eta_{3}$ increased. But for small positive values of $\operatorname{Re}\left(\eta_{3}\right)$ imaginary part $\operatorname{Im}\left(\eta_{3}\right)$ becomes effective. The most reduction on the sound transmission is seen for the negative value of $\operatorname{Im}\left(\eta_{3}\right)$.

Figure 7 shows an excellent agreement between the present paper (for the case of extended inlet length $k l \rightarrow 0$ ) and the previous study [9] of the author (for the case of 


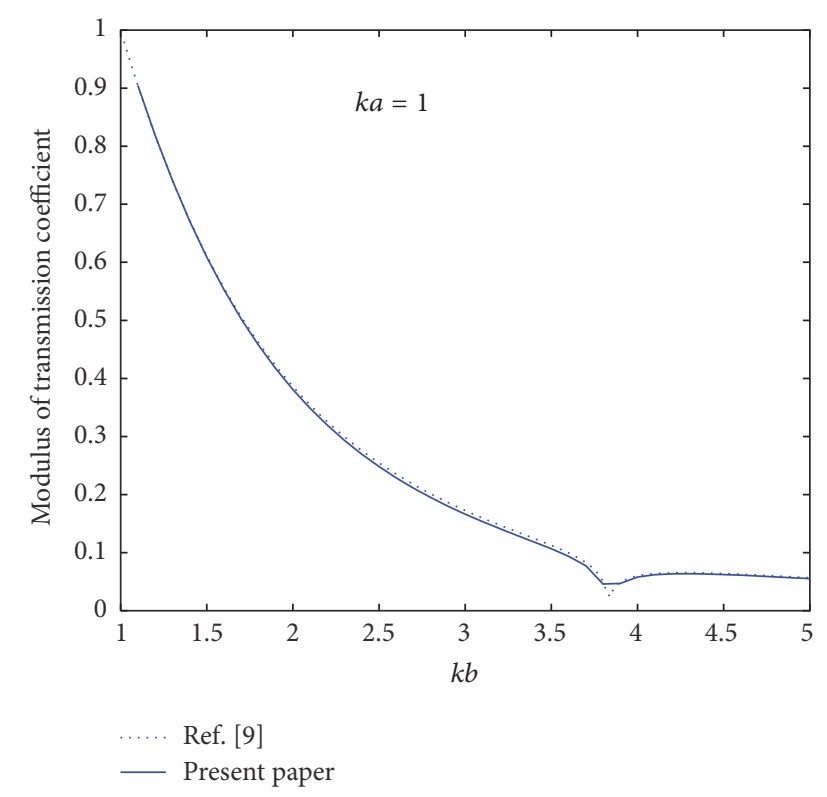

FIgURE 7: Transmission coefficient $\mathscr{T}$ versus the expansion chamber radius $k b$.

expansion chamber length $k l \rightarrow \infty$ and surface admittance is taken to be zero). In this comparison, transmission coefficient is calculated as though it is in a rigid-walled duct with sudden area expansion (without extended inlet).

\section{Conclusions}

This paper examines the transmission of sound waves in an extended tube resonator whose walls in overlapping region, where extended inlet and expanding duct walls overlap, are treated by acoustically absorbing materials of finite length. In the present work the lined region of the inner surface is assumed to be finite which makes the problem more complicated. To overcome the additional difficulty caused by the impedance discontinuity a hybrid method of formulation consisting of expressing the total field in terms of complete sets of orthogonal waveguide modes where available and using the Fourier transform elsewhere is adopted. The mixed boundary value problem is reduced to a Wiener-Hopf equation whose solution involves infinitely many expansion coefficients satisfying an infinite system of linear algebraic equations. These equations are solved numerically and the effects of various parameters on transmitted field such as the extended inlet length and the surface admittance of the lined section are displayed graphically. As a future work a similar problem now with an extended outlet will be studied following the same method used here.

\section{Competing Interests}

The author declares that there is no conflict of interests regarding the publication of this paper.

\section{References}

[1] J. W. Miles, "The analysis of plane discontinuities in cylindrical tubes. Part I," The Journal of the Acoustical Society of America, vol. 17, pp. 259-271, 1946.

[2] M. L. Munjal, Acoustics of Ducts and Mufflers, WileyInterscience, New York, NY, USA, 1987.

[3] J. Kergomard and A. Garcia, "Simple discontinuities in acoustic waveguides at low frequencies: critical analysis and formulae," Journal of Sound and Vibration, vol. 114, no. 3, pp. 465-479, 1987.

[4] A. Selamet and P. M. Radavich, "The effect of length on the acoustic attenuation performance of concentric expansion chambers: an analytical, computational and experimental investigation," Journal of Sound and Vibration, vol. 201, no. 4, pp. 407426, 1997.

[5] M. Åbom, "Derivation of four-pole parameters including higher order mode effects for expansion chamber mufflers with extended inlet and outlet," Journal of Sound and Vibration, vol. 137, no. 3, pp. 403-418, 1990.

[6] K. S. Peat, "The acoustical impedance at the junction of an extended inlet or outlet duct," Journal of Sound and Vibration, vol. 9, pp. 101-110, 1991.

[7] A. Selamet and Z. L. Ji, "Acoustic attenuation performance of circular expansion chambers with extended inlet/outlet," Journal of Sound and Vibration, vol. 223, no. 2, pp. 197-212, 1999.

[8] A. D. Rawlins, "Radiation of sound from an unflanged rigid cylindrical duct with an acoustically absorbing internal surface," Proceedings of the Royal Society. London. Series A. Mathematical, Physical and Engineering Sciences, vol. 361, no. 1704, pp. 65-91, 1978.

[9] A. Demir and A. Büyükaksoy, "Transmission of sound waves in a cylindrical duct with an acoustically lined muffler," International Journal of Engineering Science, vol. 41, no. 20, pp. 24112427, 2003.

[10] A. Büyükaksoy and A. Demir, "Diffraction of sound waves by a rigid cylindrical cavity of finite length with an internal impedance surface," Zeitschrift für Angewandte Mathematik und Physik, vol. 56, no. 4, pp. 694-717, 2005.

[11] A. Büyükaksoy, G. Uzgören, and F. Birbir, “The scattering of a plane wave by two parallel semi-infinite overlapping screens with dielectric loading," Wave Motion, vol. 34, no. 4, pp. 375389, 2001.

[12] A. D. Rawlins, "A bifurcated circular waveguide problem," IMA Journal of Applied Mathematics, vol. 54, no. 1, pp. 59-81, 1995. 


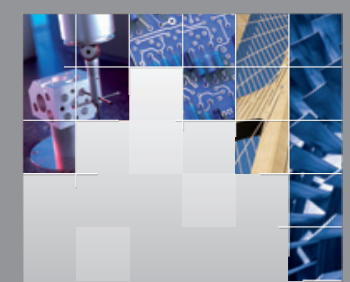

\section{Enfincering}
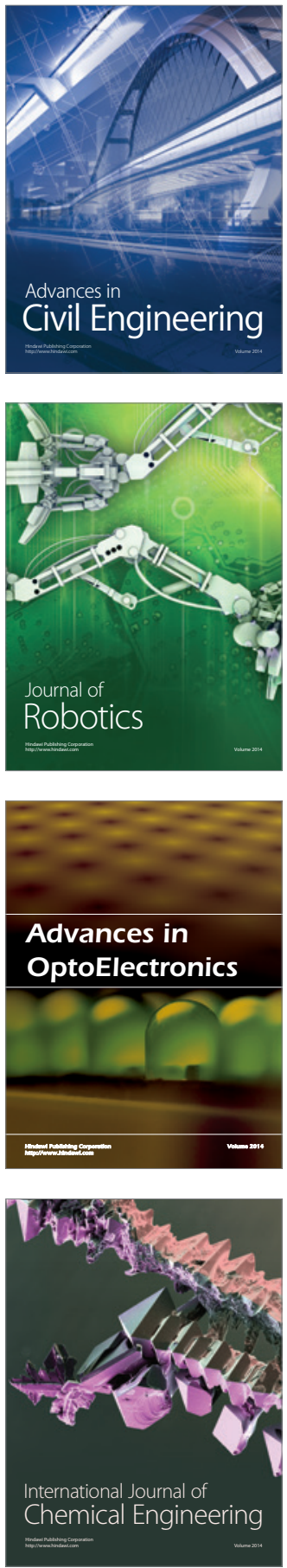

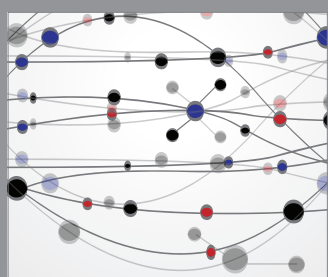

The Scientific World Journal

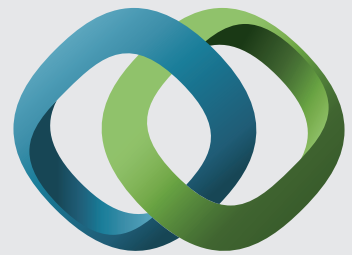

\section{Hindawi}

Submit your manuscripts at

http://www.hindawi.com
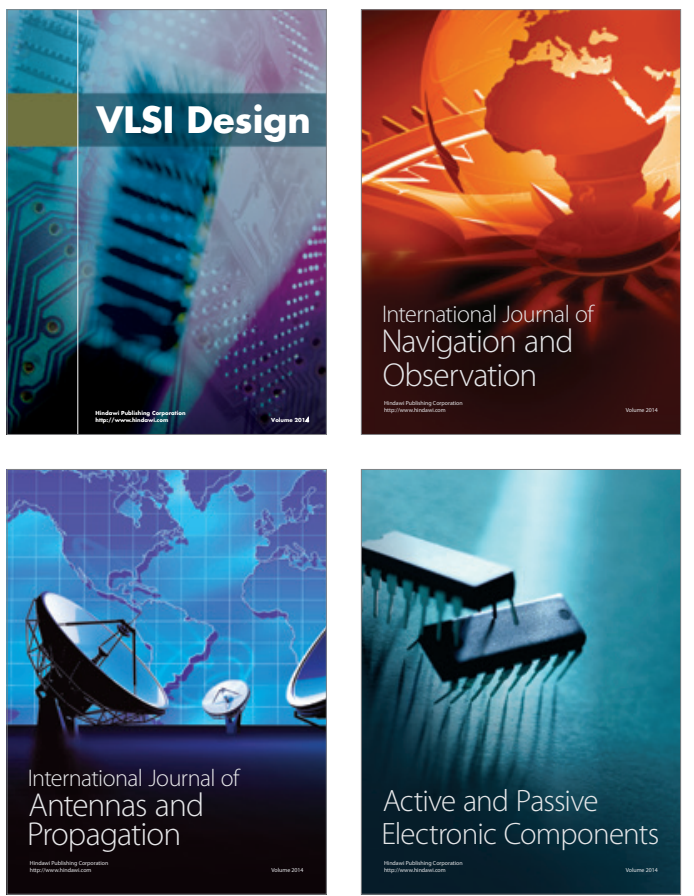
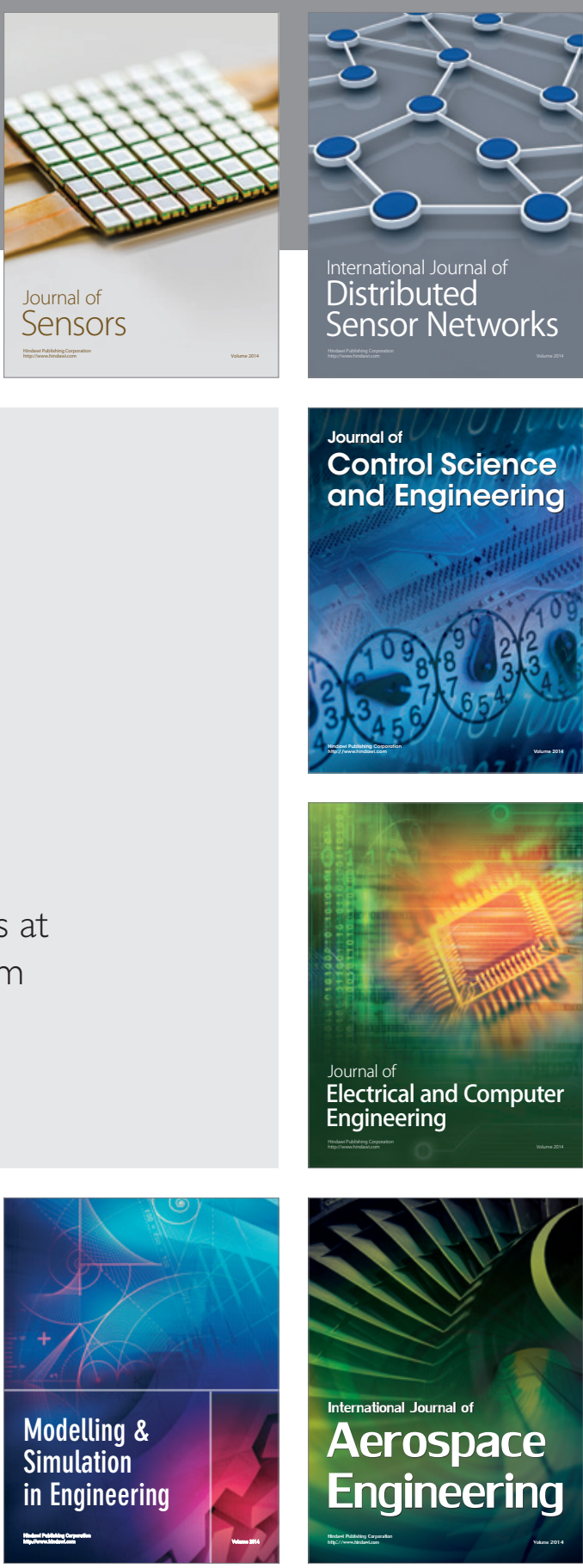

International Journal of

Distributed

Sensor Networks

Journal of

Control Science

and Engineering
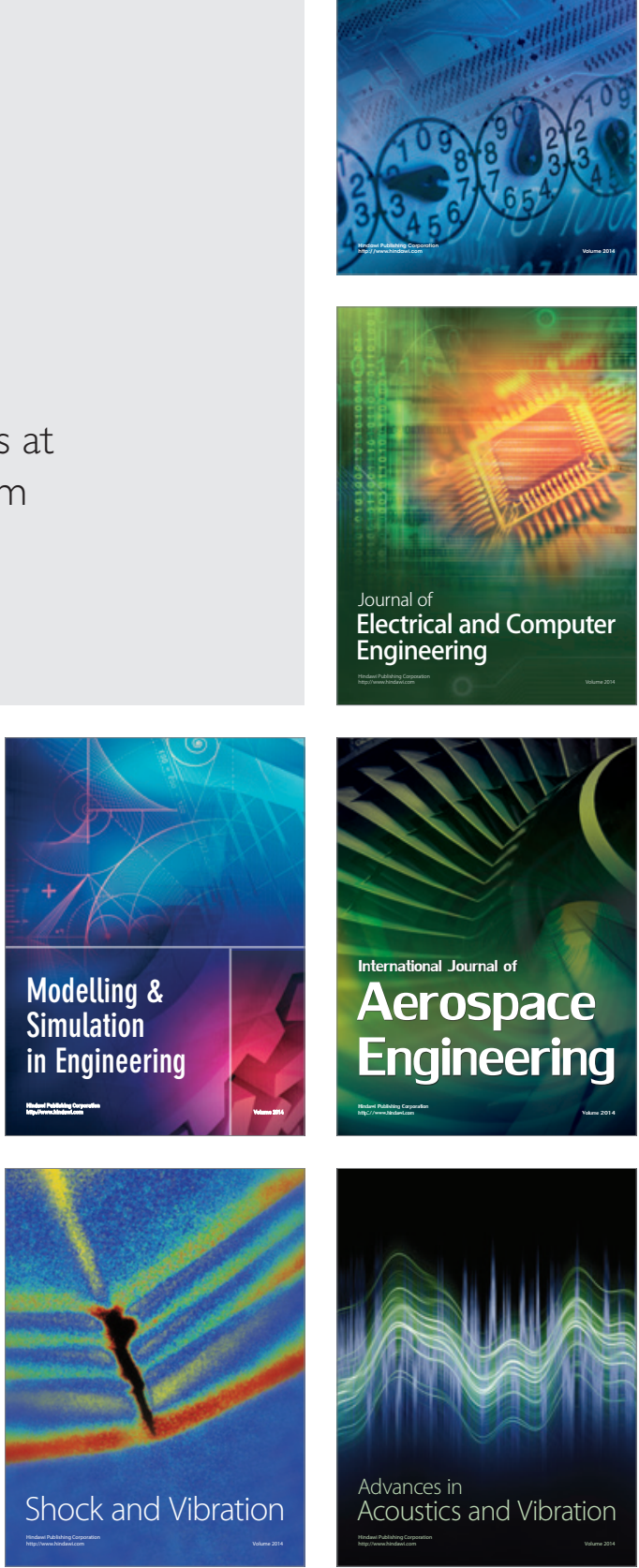